\title{
Social and Behavioral Risk Marker Clustering Associated with Biological Risk Factors for Coronary Heart Disease: NHANES 2001-2004
}

\author{
Nicholas J. Everage, ${ }^{1}$ Crystal D. Linkletter, ${ }^{2,3}$ Annie Gjelsvik, ${ }^{1,4}$ \\ Stephen T. McGarvey, ${ }^{1,5}$ and Eric B. Loucks ${ }^{1,6}$ \\ ${ }^{1}$ Department of Epidemiology, Brown University School of Public Health, Providence, RI 02912, USA \\ ${ }^{2}$ Center for Statistical Sciences, Brown University School of Public Health, Providence, RI 02912, USA \\ ${ }^{3}$ Department of Biostatistics, Brown University School of Public Health, Providence, RI 02912, USA \\ ${ }^{4}$ Diabetes Prevention and Control Program, Rhode Island Department of Health, Providence, RI 02912, USA \\ ${ }^{5}$ International Health Institute, Brown University School of Public Health, Providence, RI 02912, USA \\ ${ }^{6}$ Center for Population Health \& Clinical Epidemiology, Brown University School of Public Health, Providence, RI 02912, USA
}

Correspondence should be addressed to Nicholas J. Everage; nickeverage@gmail.com

Received 30 April 2013; Revised 20 November 2013; Accepted 24 December 2013; Published 25 February 2014

Academic Editor: Kazuhiko Kotani

Copyright ( 2014 Nicholas J. Everage et al. This is an open access article distributed under the Creative Commons Attribution License, which permits unrestricted use, distribution, and reproduction in any medium, provided the original work is properly cited.

Background. Social and behavioral risk markers (e.g., physical activity, diet, smoking, and socioeconomic position) cluster; however, little is known whether clustering is associated with coronary heart disease (CHD) risk. Objectives were to determine if sociobehavioral clustering is associated with biological CHD risk factors (total cholesterol, HDL cholesterol, systolic blood pressure, body mass index, waist circumference, and diabetes) and whether associations are independent of individual clustering components. Methods. Participants included 4,305 males and 4,673 females aged $\geq 20$ years from NHANES 2001-2004. Sociobehavioral Risk Marker Index (SRI) included a summary score of physical activity, fruit/vegetable consumption, smoking, and educational attainment. Regression analyses evaluated associations of SRI with aforementioned biological CHD risk factors. Receiver operator curve analyses assessed independent predictive ability of SRI. Results. Healthful clustering (SRI $=0)$ was associated with improved biological CHD risk factor levels in 5 of 6 risk factors in females and 2 of 6 risk factors in males. Adding SRI to models containing age, race, and individual SRI components did not improve C-statistics. Conclusions. Findings suggest that healthful sociobehavioral risk marker clustering is associated with favorable CHD risk factor levels, particularly in females. These findings should inform social ecological interventions that consider health impacts of addressing social and behavioral risk factors.

\section{Introduction}

Coronary heart disease (CHD) is the leading cause of death in the United States, despite extensive gains in primary and secondary prevention $[1,2]$. Social factors, including education, may be important risk markers for CHD [36]. Better educated and wealthier groups have achieved significant decreases in CHD risk factors compared to their less educated, poorer counterparts [7]. Furthermore, there has been emphasis on social ecological intervention models, that take into account the social context such as socioeconomic position (e.g., education), race/ethnicity, neighborhood characteristics and social network transmission of health behaviors, which may shape the success of health behavior interventions or the behaviors themselves [8-12]. Also, lifestyle risk factors, such as smoking, low physical activity, low fruit/vegetable intake, low fiber intake, and high trans-fat and saturated fat diets have evidence to be responsible for a substantial proportion of CHD events [13-19]. While a number of individual- and community-based trials, 
including the Stanford Five-City Project, the Pawtucket Heart Health Program, and the Multiple Risk Factor Intervention Trial (MRFIT), have attempted to change lifestyle risk factors/behaviors related to CHD [20-25], systematic reviews on cardiovascular disease prevention interventions have shown little to modest favorable reductions in cardiovascular disease risk in response to these programs $[25,26]$. One method for improving interventions may be focusing on multiple social and behavioral risk factors at a time. Previous work has shown that social and behavioral risk markers (including physical activity, diet, smoking and educational attainment) cluster [27], but it is not known whether the clustering is related to $\mathrm{CHD}$ risk or whether any associations with $\mathrm{CHD}$ risk are independent of the individual social and behavioral risk marker components. In considering interventions to prevent $\mathrm{CHD}$, it may be helpful to consider the potential mutually reinforcing characteristics of both social and behavioral risk markers. This could facilitate the creation of more effective interventions, for example, if interventions on a single risk marker (e.g., physical activity) were substantially affected by other co-occurring risk factors such as diet, smoking and socioeconomic position. Understanding which social and behavioral risk factors might mutually influence each other could substantially inform etiologic understanding of $\mathrm{CHD}$, and identify possible interventions aimed at addressing the mutually reinforcing causes of CHD. Comparable research on the metabolic syndrome, another clustering of conditions relevant to $\mathrm{CHD}$ and diabetes, has demonstrated that while the biological CHD risk factors (e.g., blood pressure, central obesity, fasting glucose and lipids) do co-occur more often than would be expected due to chance (i.e., cluster), the clustering does not appear to confer risk above its individual components [28-33]. Therefore, the objective of this study was to evaluate whether sociobehavioral clustering is associated with biological CHD risk factors including hypertension, dyslipidemia, obesity, and diabetes in the National Health and Nutrition Examination Survey (NHANES) 2001-2004. In addition, we aimed to determine if the clustering itself is associated with biological CHD risk factors independently of the individual contributions of each social and behavioral risk marker.

\section{Methods}

2.1. Study Sample. The study included participants from the 2001-2002 and 2003-2004 NHANES. Participants were interviewed in their homes and in mobile examination centers across the United States. The study sample for this analysis included all participants $\geq 20$ years old $(N=10,452)$. Participants missing data on education $(n=22)$, smoking status $(n=35)$, physical activity $(n=306)$, and/or fruit and vegetable intake $(n=1,260)$ were excluded from analyses. The high number of missing fruit and vegetable intake data was primarily due to participants either not completing dietary recalls due to refusal, having incomplete information on diet, or arriving late to the NHANES mobile examination centers with resulting insufficient time to complete dietary assessments. The final analytic sample size was 8,798. All participants had at least one criteria for determining diabetes disease status. However, there were missing data on body mass index $(n=261)$, total cholesterol $(n=444)$, high-density lipoprotein cholesterol (HDL) $(n=445)$, and systolic blood pressure $(n=371)$. The dependent variable with the median amount of missing data was systolic blood pressure. Therefore, we included this variable in our comparison analysis of included and excluded participants. Compared to excluded participants, included participants were younger (49 versus 56 years; $P<0.0001$ ), had slightly higher smoking prevalence $(22.6 \%$ versus $19.5 \%$ smokers; $P=0.004)$, were less likely to have attended college $(53.1 \%$ versus $61.7 \%$ with $>$ high school education; $P<0.0001)$, and were more likely to meet physical activity guidelines (52.9\% versus $44.0 \%$ met guidelines; $P<0.0001)$. Included participants were more likely to be non-Hispanic white (53.3\% versus 52.1\%) or Mexican American/other Hispanic race/ethnicity, and less likely to be non-Hispanic black $(18.9 \%$ versus $20.8 \%$ ) or "other" race/ethnicity (3.7\% versus $5.3 \%$; $P=0.0007)$. Included participants had slightly lower systolic blood pressure (125.9 versus $130.8 \mathrm{mmHg} ; P<0.0001$ ), waist circumference ( $97.3 \mathrm{~cm}$ versus $98.7 \mathrm{~cm} ; P=0.03)$, HDL (53.4 versus $54.5 \mathrm{mg} / \mathrm{dL} ; P=0.05)$, less obesity (31.5\% versus $34.6 \% ; P=0.05)$ and less likely to have diabetes $(11.6 \%$ versus $14.2 \% ; P=0.002$ ) than excluded participants; however there were no differences in total cholesterol (203.1 versus $201.2 \mathrm{mg} / \mathrm{dL} ; P=0.20$ ), or fruit and vegetable guideline compliance $(53.7 \%$ versus $56.1 \%$ met guidelines; $P=0.26)$.

\subsection{Biological Coronary Heart Disease (CHD) Risk Factors}

2.2.1. Lipids. Total cholesterol was determined enzymatically using the Trinder-type method [34]. HDL cholesterol was assessed using standard enzymatic assays; molecules were separated from plasma by chemical precipitation with dextran sulfate-magnesium, and the resulting supernatant was assayed for cholesterol $[35,36]$. The NHANES quality control and quality assurance protocols met the 1988 Clinical Laboratory Improvement Act mandates. Participants were classified as having dyslipidemia according to National Cholesterol Education Program (NCEP) defined cut-points for HDL levels of $<40 \mathrm{mg} / \mathrm{dL}$ in males, $<50 \mathrm{mg} / \mathrm{dL}$ in females, and total cholesterol levels $\geq 240 \mathrm{mg} / \mathrm{dL}$ in males and females [37].

2.2.2. Systolic Blood Pressure. Certified physician examiners conducted three blood pressure measurements after having participants rest in a seated position for 5 minutes [38]. The training of physician examiners as well as extensive documentation on the quality control measures are found in the NHANES physical examination protocol [38]. The mean of the second and third systolic blood pressure measurements was used for analyses. Participants with systolic blood pressure $\geq 140 \mathrm{mmHg}$ were classified as having hypertension according to the 2003 Seventh Report of the Joint National Committee on Prevention, Detection, Evaluation, and Treatment of High Blood Pressure [39]. 
2.2.3. Obesity. Obesity was determined by body mass index (BMI) and waist circumference. Body weight was measured to the nearest $0.1 \mathrm{lb}$ with the use of a daily calibrated Toledo digital scale and with subjects wearing only disposable paper gowns and slippers [40]. This measurement was then converted to kilograms. Height was measured with the use of a stadiometer (to the nearest 0.25 in, and then converted to meters) [40]. BMI was calculated as the weight in kilograms divided by the square of the height in meters $\left(\mathrm{kg} / \mathrm{m}^{2}\right)$. Participants with a BMI $\geq 30 \mathrm{~kg} / \mathrm{m}^{2}$ were classified as obese. With participants standing and having normally expelled air, trained examiners measured participants' waist circumference using a tape measure placed at the lateral border of the ilium [40]. Participants who exceeded waist circumference guidelines (males: $>94 \mathrm{~cm}$; females: $>80 \mathrm{~cm}$ ) were considered to have large waist circumference [41].

2.2.4. Diabetes. Presence of diabetes was defined as selfreported, doctor-diagnosed diabetes; self-reported use of oral glucose-lowering medications; self-reported insulin use; visually confirmed pharmaceutical use of oral glucoselowering drug and insulin; or fasting plasma glucose $\geq 126 \mathrm{mg} / \mathrm{dL}$. Plasma glucose was collected from participants after a 12-hour fast before the examination. Glucose levels were measured using the hexokinase ultraviolet method [42]. The NHANES quality control and quality assurance protocols met the 1988 Clinical Laboratory Improvement Act mandates.

\subsection{Social and Behavioral Risk Marker Clustering Variables.}

The social independent variable (education) and the three behavioral independent variables (diet, physical activity, smoking) were selected a priori. Physical activity, diet and smoking were selected due to evidence that they are three of the most important behavioral risk factors for CHD [1517]. With regard to social factors, socioeconomic position was selected as it has been demonstrated to be consistently associated with $\mathrm{CHD}$ in systematic reviews [18]. Of the different measures of SEP (e.g., income, occupation, education), we selected education as it remains stable across adulthood, participants are more likely to agree to report on it compared to income for which there are more missing data, and it is a variable that can influence other SEP measures such as income and occupation [19].

2.3.1. Physical Activity. The relevant national physical activity guidelines for the period from 2001-2004 were those by the Centers for Disease Control and the American College of Sports Medicine [43]. The guidelines stated that "adults should accumulate 30 minutes or more of moderate-intensity physical activity on most, preferably all, days of the week" [43]. Due to a lack of specificity in the guidelines language, we interpreted the recommendations as suggesting that adults should exercise at least 5 days per week for at least 30 minutes, or $\geq 150$ minutes of moderate and/or vigorous of physical activity per week [44]. Estimates of weekly physical activity were based on participant responses to the following questions: "Over the past 30 days, have you walked or bicycled as part of getting to and from work, or school, or to do errands?" and "Over the past 30 days, did you do any tasks in or around your home or yard for at least 10 minutes that required moderate or greater physical effort [45]?" Participants also reported time and effort spent on 45 leisure-time activities, such as gardening, weightlifting, walking, swimming, and yoga [46]. The average duration and frequency for each activity was reported and used to estimate the weekly physical activity time for each participant.

2.3.2. Fruit and Vegetable Intake. Fruit and vegetable consumption in NHANES was measured using 24-hour dietary recalls $[47,48]$. The recalls follow methodology based on the joint-venture program "What We Eat in America" established by the U.S. Department of Agriculture (USDA) and the Department of Health and Human Services [49]. From 2001-2002, NHANES collected one 24-hour recall, but in 2003-2004 expanded collection to one 24-hour recall in the Mobile Examination Center and an additional recall 3-10 days later. In 2003-2004, approximately $87 \%$ of the sample completed both dietary recalls. Based on the dietary recall, the USDA calculated the number of cups of each food group consumed by the participant using the MyPyramid Equivalents Database (MPEDS). The 1992 Food Guide Pyramid recommendations were still in effect during the 2001-2004 time period of the analysis. Therefore, we considered the participants as having met the guidelines if they consumed $\geq 3$ servings ( 1.5 cups) of vegetables and $\geq 2$ servings ( 1 cup) of fruit per day [50].

2.3.3. Smoking Status. Participants reporting smoking $<100$ cigarettes in their life or no longer smoking were considered nonsmokers. We classified participants who reported smoking "every day" or "some days" as current smokers.

2.3.4. Socioeconomic Position. Participants reported their highest level of educational attainment and, based on previous literature $[51,52]$, we operationalized the variable as $\leq$ high school (i.e., high school diploma, General Equivalency Diploma (GED), or less) versus > high school (e.g., some college, associate's degree, college or postgraduate).

2.4. Covariates. Participants reported their age, sex, and race/ethnicity during the home visit of NHANES. The categories for race/ethnicity were: non-Hispanic black $(n=1,719)$, non-Hispanic white $(n=4,752)$, Mexican-American/other Hispanic $(n=2,174)$, or "other race" $(n=333)$. Patients also reported if they were currently taking antihypertensive medications and/or cholesterol-lowering medication.

2.5. Statistical Analyses. Each of the sociobehavioral clustering variables (i.e., smoking status, meeting fruit and vegetable and physical activity guidelines, and education attainment) were dichotomized into indicator variables (i.e., $1=$ Yes, 0 $=$ No) using cut points described above. A Sociobehavioral Risk Marker Index (SRI) ranging from $0-4(0=$ No risk factors; $4=$ All risk factors) was constructed by summing 
the indicator variables. Statistically significant clustering has been identified elsewhere using the SRI [27].

Multivariable-adjusted linear and logistic regression analyses compared SRI $=0$ and SRI $=4$ to having 1,2 or 3 risk markers $(\mathrm{SRI}=1-3)$, in order to evaluate associations of healthful (SRI $=0)$ and unhealthful $(S R I=4)$ risk marker clustering with biological CHD risk factors. Similar analyses have been conducted to assess the risk conferred by the metabolic syndrome over its individual components with incident CHD outcomes (e.g., CHD-related mortality) [30, 31]. Because the NHANES data are cross-sectional and lack incident $\mathrm{CHD}$ information, analyses used C-statistics from receiver operating curves (ROC) to evaluate the predictive ability (i.e., comparison of the sensitivity and false positive proportions) of the of the SRI on the CHD biological risk factors [30,31]. Sex-, racial/ethnic- and age-stratified analyses were used to understand potential effect modification of the associations between the SRI and the CHD biological risk factors. Formal statistical testing for interactions demonstrated significant interactions between the SRI $=0$ (versus $1,2,3$ ) and sex for 3 of the $6 \mathrm{CHD}$ biological risk factor outcomes, specifically $\operatorname{HDL}(P=0.002)$, waist circumference $(P<$ $0.0001)$ and obesity $(P<0.0001)$. Statistically significant interactions were also found between SRI $=4$ (versus 1,2 , $3)$ and sex for $\operatorname{HDL}(P=0.003)$ and waist circumference $(P=0.04)$. Consequently analyses were performed sexspecific. With regard to race/ethnicity, only one of twelve statistical tests (interaction with $\operatorname{HDL}(P=0.03)$; for the 6 biological CHD risk factors in males and females separately) for interactions between SRI and race/ethnicity were significant $(P<0.05)$, consequently race/ethnicity-specific analyses were not performed. All analyses were adjusted for race/ethnicity. Statistical analyses were conducted using SAS 9.2 (Carey, NC).

\section{Results}

Characteristics of the study sample are shown in Table 1 and the distribution of the SRI by sex is described in Figure 1. Females demonstrated higher BMI, total cholesterol, and HDL cholesterol, but lower smoking, systolic blood pressure, waist circumference, cholesterol medication use, physical activity guideline compliance and fruit/vegetable consumption guideline compliance than males. There were no significant differences between sexes for diabetes, antihypertensive medication use, education, race/ethnicity or age.

The individual components of the SRI (i.e., education, smoking, fruit and vegetable consumption and physical activity) were associated with the biological CHD risk factors fairly extensively in females and less so in males (Table 2). Specifically, of the 6 biological CHD risk factors that were evaluated (i.e., systolic blood pressure, total cholesterol, HDL cholesterol, waist circumference, body mass index and diabetes), the SRI components education, smoking, fruit/vegetable consumption and physical activity were associated with 5,1 , 3 and 4 of the biological CHD risk factors, respectively, in females, and were associated with only 2, 2, 0 and 3 of the biological CHD risk factors, respectively, in males (Table 2).

Healthful clustering of SRI components was strongly associated with more favorable biological CHD risk factors in females ( 5 of 6 CHD risk factors were associated with SRI $=0$ versus $\mathrm{SRI}=1-3)$ and less so in males ( 2 of 6 risk factors were associated with SRI $=0$ versus SRI $=1-3$; Table 3 ). In an effort to evaluate if the clustering itself was associated with the biological CHD risk factors over and above the individual contributions of SRI components, analyses additionally adjusted for the individual SRI components, and generally showed substantial reductions in effect size, suggesting that the clustering itself was not overarchingly associated with CHD risk factors independently of the individual SRI components (Table 3). Unhealthy clustering of SRI components (i.e., SRI $=4$ ) was not substantially associated with CHD risk factors in females or males (Table 3 ).

ROC curve analyses utilizing C-statistics demonstrated predictive ability for SRI in relation to all biological CHD risk factors, although the SRI predictive ability was particularly high for systolic hypertension and diabetes (Table 4). In an effort to evaluate if the clustering associated with SRI $=4$ or SRI $=0$ contributed to biological CHD risk factors levels over and above the individual SRI components themselves (i.e., education, smoking, fruit/vegetable consumption and physical activity), three different C-statistics were calculated that included age, race/ethnicity and (1) individual SRI components only, (2) SRI only, or (3) both SRI and individual SRI components. As shown in Table 4, generally there was very minimal change in C-statistic values in models that included both SRI and the individual SRI components, compared with models that included only the SRI, or the individual SRI components only (in addition to age and race/ethnicity). These findings suggested that the SRI clustering itself did not contribute to biological CHD risk factor levels over and above age, race/ethnicity and the individual SRI components. Similarly, the individual SRI components did not contribute to biological CHD risk factor levels independently of age, race/ethnicity and the SRI clustering values of SRI $=0$ or SRI $=4$, versus SRI $=1-3$.

\section{Discussion}

Overall findings demonstrated that healthful SRI clustering was associated with improved biological CHD risk factor (systolic blood pressure, HDL cholesterol, waist circumference, BMI and diabetes) levels in females, and less so in males (healthful SRI clustering associated only with HDL cholesterol and diabetes). Unhealthy SRI clustering generally was not associated with biological CHD risk factors. Furthermore, findings suggested that the SRI clustering does not affect biological CHD risk factors independent of its individual components.

4.1. Prior Research. To our knowledge, no other studies have evaluated associations of social and behavioral risk marker clustering with biological CHD risk factors. However, other measures of biological CHD risk factor clustering, specifically 
TABLE 1: Descriptive characteristics of study participants stratified by sex, NHANES 2001-2004.

\begin{tabular}{|c|c|c|c|c|}
\hline & \multicolumn{2}{|c|}{$\operatorname{Men}(n=4,305)$} & \multicolumn{2}{|c|}{ Women $(n=4,673)$} \\
\hline & $\begin{array}{c}\text { Mean or } \\
\text { proportion }\end{array}$ & $95 \% \mathrm{CI}^{*}$ & $\begin{array}{l}\text { Mean or } \\
\text { proportion }\end{array}$ & $95 \% \mathrm{CI}$ \\
\hline \multicolumn{5}{|l|}{ Age } \\
\hline $20-29$ years, $\%$ & 17.7 & $15.0-20.4$ & 20.5 & $18.0-23.1$ \\
\hline $30-39$ years, $\%$ & 15.7 & $13.0-18.4$ & 18.4 & $15.8-21.0$ \\
\hline $40-49$ years, $\%$ & 18.0 & $15.3-20.7$ & 16.4 & $13.8-19.0$ \\
\hline $50-59$ years, $\%$ & 13.7 & $10.9-16.5$ & 12.1 & $9.4-14.8$ \\
\hline $60-69$ years, $\%$ & 14.7 & $12.0-17.5$ & 14.7 & $12.0-17.3$ \\
\hline $70-79$ years, $\%$ & 12.7 & $9.9-15.5$ & 9.9 & $7.1-12.6$ \\
\hline$\geq 80$ years, $\%$ & 7.4 & $4.6-10.3$ & 8.0 & $5.3-10.8$ \\
\hline \multicolumn{5}{|l|}{ Race/ethnicity } \\
\hline Non-Hispanic white, $\%$ & 53.0 & $50.9-55.0$ & 52.9 & $50.9-54.9$ \\
\hline Non-Hispanic black, \% & 19.2 & $16.5-21.9$ & 19.1 & $16.5-21.7$ \\
\hline Mexican-American/other Hispanic, \% & 24.4 & $21.8-27.0$ & 24.0 & $21.5-26.5$ \\
\hline Other race, $\%$ & 3.4 & $0.5-6.4$ & 4.0 & $1.2-6.8$ \\
\hline \multicolumn{5}{|l|}{ Education } \\
\hline$\leq$ High school diploma/GED, $\%$ & 54.2 & $52.1-56.2$ & 52.5 & $50.5-54.5$ \\
\hline >High school diploma/GED, \% & 45.8 & $43.6-48.0$ & 47.5 & $45.4-49.6$ \\
\hline Current smoker, \% & 27.2 & $24.6-29.7$ & 18.4 & $15.8-21.0$ \\
\hline Did not meet fruit and vegetable guidelines, $\%^{\dagger}$ & 51.7 & $49.6-53.8$ & 55.4 & $53.5-57.3$ \\
\hline Did not meet physical activity guidelines, $\%^{\ddagger}$ & 43.8 & $41.5-46.0$ & 51.2 & $49.2-53.2$ \\
\hline Current diabetes, $\%^{* *}$ & 12.7 & $9.9-15.5$ & 10.9 & $8.2-13.6$ \\
\hline Systolic blood pressure, $\mathrm{mmHg}$ & 126.7 & $126.2-127.3$ & 125.0 & $124.4-125.7$ \\
\hline Taking antihypertensive medication, $\%$ & 22.4 & $19.8-25.1$ & 23.6 & $21.1-26.1$ \\
\hline Total cholesterol, mg/dL & 200.0 & 198.5-201.3 & 206.1 & $204.8-207.4$ \\
\hline HDL cholesterol, mg/dL & 47.5 & $47.1-47.9$ & 58.9 & $58.4-59.4$ \\
\hline Taking cholesterol-lowering medication, $\%$ & 20.5 & $17.2-23.7$ & 16.4 & $13.2-19.5$ \\
\hline Body mass index $\left(\mathrm{kg} / \mathrm{m}^{2}\right)$ & 27.9 & $27.7-28.1$ & 28.7 & $28.5-28.9$ \\
\hline Waist circumference $(\mathrm{cm})$ & 99.7 & $99.3-100.1$ & 95.5 & $95.0-95.9$ \\
\hline
\end{tabular}

the metabolic syndrome, have been well demonstrated to be related to both incident $\mathrm{CHD}$ and diabetes [30-32]. However, similarly to the sociobehavioral clustering described here, the metabolic syndrome does not appear to predict CHD risk independently of its individual components $[30,31,53,54]$.

4.2. Potential Mechanisms. The SRI components (i.e., education, smoking, fruit and vegetable consumption and physical activity) were generally related to CHD biological risk factors. Specifically, education was generally inversely associated with systolic blood pressure $[5,7,55-58]$, waist circumference [59], body mass index $[55,56]$ and diabetes $[7,60]$, positively associated with HDL cholesterol $[61,62]$, and not related to total cholesterol [7, 62]. Furthermore, smoking was overall positively associated with systolic blood pressure [63], total cholesterol $[64,65]$, waist circumference [66], and diabetes $[67,68]$, and inversely associated with HDL [65]. It should be noted that those who smoke heavily are generally more likely to be obese, but light and moderate smokers have decreased risk of obesity [66]. Studies have demonstrated that fruit and vegetable intake is typically inversely associated with systolic blood pressure in men and women $[69,70]$ and waist circumference in women [71], but evidence suggests a lack of association of fruit and vegetable consumption with total cholesterol [72] and diabetes [73-75]. Research is also equivocal on fruit and vegetable intake's effect on HDL and obesity [71, 76-79]. The weakness or lack of association between fruit and vegetable intake and blood lipid levels may reflect the need to account for other aspects of diet, including healthful oil and fiber consumption [77, 80-82]. Finally, physical activity has been inversely associated with systolic 


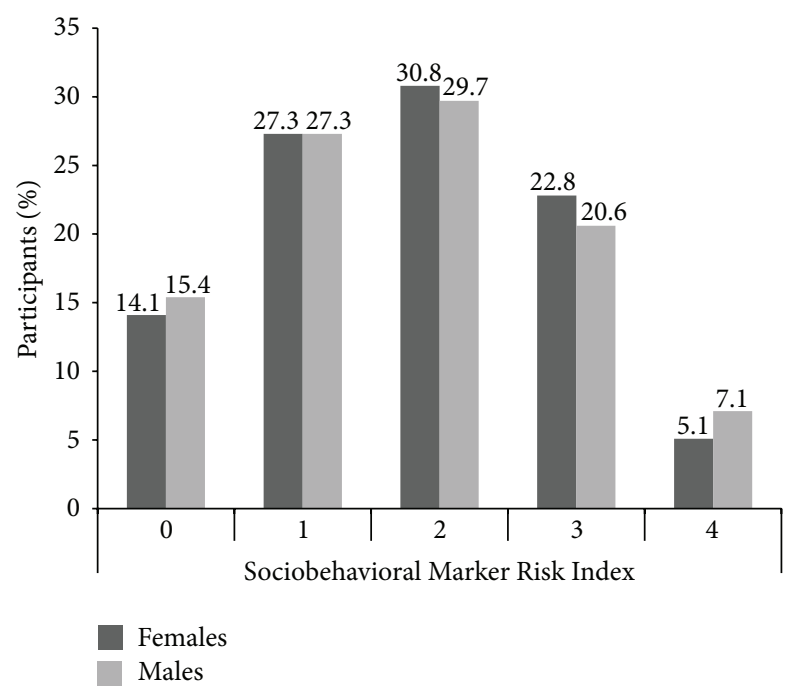

FIgURE 1: Distribution of Sociobehavioral Risk Marker Index (SRI) by Sex, NHANES 2001-2004.

blood pressure [83, 84], waist circumference [85, 86], obesity [87-89], and diabetes [90, 91]; and positively associated with HDL $[82,92]$. Evidence for an inverse effect between physical activity and total cholesterol is not consistent $[82,93]$.

Gender differences were observed, where in females the SRI was associated with systolic blood pressure, HDL cholesterol, waist circumference, BMI and diabetes, while in males the SRI was associated with HDL cholesterol and diabetes. Similar gender differences have been seen in the educationhealth literature where associations of education with CHD, diabetes and metabolic syndrome are often stronger in females than males [18, 94-97]. Potential mechanisms for gender differences include obesity-related effects on social mobility and greater concurrent psychosocial risk for low socioeconomic position women than men. For example, obesity was reported to be stigmatized more highly in women than men, and obesity may limit upward social mobility more so in women than men [98]. Furthermore, findings demonstrate that women with low education level have additional psychosocial risks (including high depressive symptoms, income under the poverty threshold, unemployment, and single parenting) than men with low education [56]. This greater psychosocial burden in low socioeconomic position women may be associated in turn with poorer metabolic outcomes, as suggested in other studies that showed a relation between depression and metabolic syndrome $[99,100]$ and income and metabolic syndrome $[101,102]$. Future research focused on identifying mechanisms responsible for gender differences in the relation between sociobehavioral clustering and CHD risk will provide better knowledge of the potential pathways.

There have been substantial advancements emphasizing the importance of social ecological intervention models that take into account the social context which may shape the success of health behavior interventions, or the behaviors themselves $[8-10,12,103]$. In considering interventions to prevent CHD, it may be helpful to consider the mutually reinforcing characteristics of both social and behavioral risk factors. This could help to create more effective interventions. For example, if interventions on a single risk factor (e.g., physical activity) may be substantially affected by co-occurring other risk factors such as diet, smoking and socioeconomic position. Furthermore, as social and behavioral risk marker clustering has been demonstrated to occur [27], it is also important to determine whether that clustering confers risk for CHD. This study demonstrated that healthful sociobehavioral clustering was associated with biological CHD risk factors particularly in females, however the clustering itself did not confer risk over and above the individual social and behavioral risk markers themselves. Despite a lack of additional risk beyond its individual components, the SRI may still provide value to clinicians and public health researchers. Specifically, sociobehavioral clustering occurs, which may be important when devising interventions to prevent or treat the effects of the individual SRI components (education, fruit and vegetable intake, physical activity and smoking). Furthermore, healthful sociobehavioral clustering is related to improved biological CHD risk factor levels, particularly in females. This improved biological CHD risk factor levels are likely due to the individual contributions of education, physical activity, fruit and vegetable consumption and lack of smoking.

4.3. Limitations. This study assessed the risk conferred by the SRI using biological CHD risk factors, which are estimates of future risk for CHD. Future studies should evaluate associations of the SRI with clinical endpoints such as incident myocardial infarction, type 2 diabetes or mortality to better understand the importance of sociobehavioral CHD risk factor clustering with health outcomes [30, 31]. Furthermore, our study used cross-sectional data, which limits causal inference for the relation between sociobehavioral clustering and biological CHD risk factors. Additionally, recall of physical activity and fruit and vegetables intake has substantial 


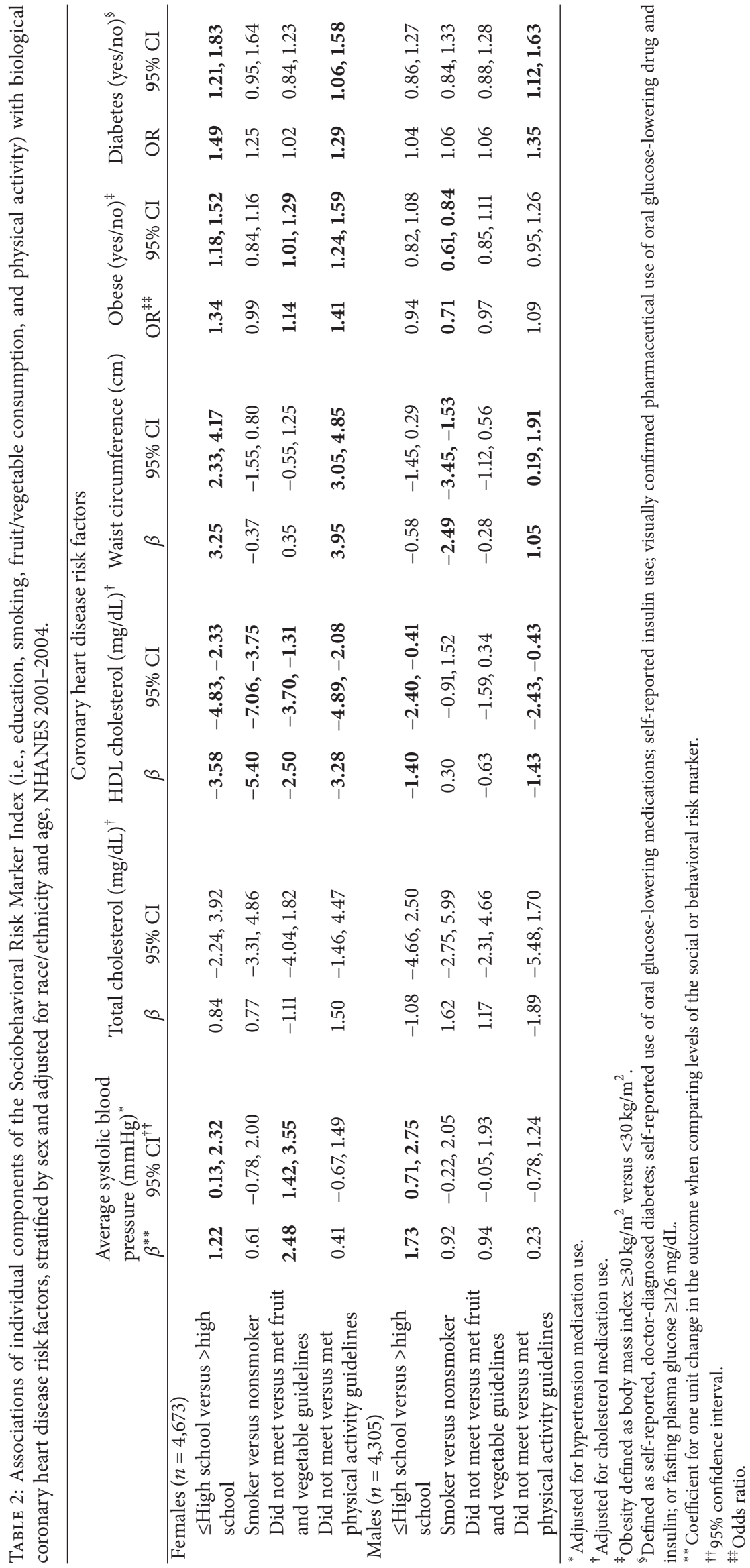




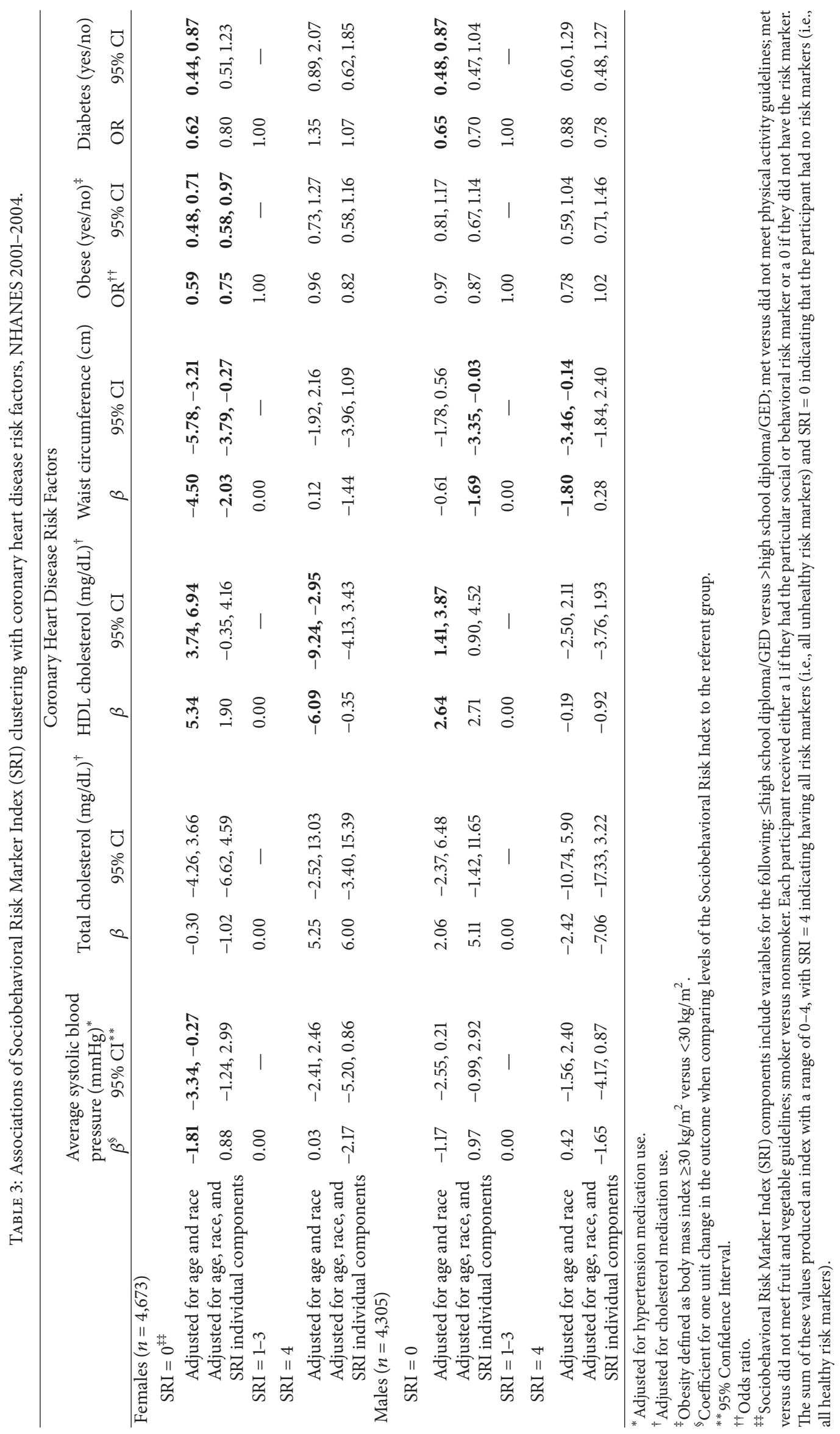




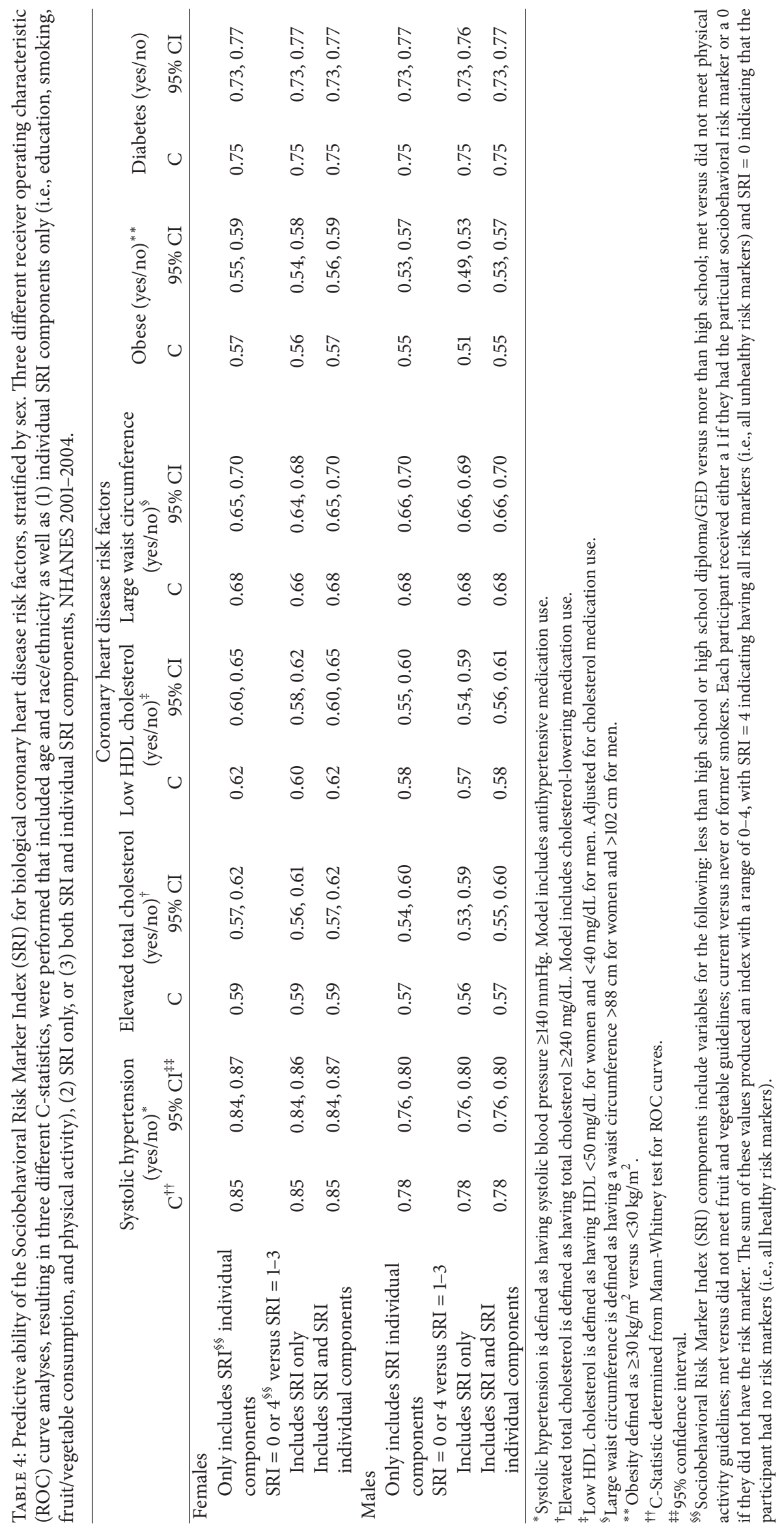


measurement error, suggesting that a reasonable amount of misclassification occurred [104]. Multiple 24-hour recalls are preferred over using dietary data from a single 24-hour period. In our study, NHANES increased the number of dietary recall days from 1 day in 2001-2002 to 2 days in 20032004. A strength of this study was the use of NHANES data, with its high level of quality control and quality assurance [105].

\section{Conclusion}

This study suggests that, particularly among women, healthful sociobehavioral risk marker clustering is related to having more favorable levels of CHD risk factors. Sociobehavioral clustering itself was not related to $\mathrm{CHD}$ risk independent of the individual clustering components, suggesting it may be the individual sociobehavioral risk factors themselves (i.e., physical activity, diet, smoking and education) that are responsible for associations with CHD risk. As social ecological intervention models advance, and take into account the social context which may shape the success of health behavior interventions $[8-10,12,103]$, it will be important to carefully consider the mutually reinforcing characteristics of both social and behavioral risk factors. This should help to create more effective interventions. As social and behavioral risk marker clustering has recently been demonstrated to occur [27], it is also important to determine whether that clustering confers risk for CHD. The current study's findings should further inform social ecological interventions that consider the potential health impacts of addressing both social and behavioral risk factors.

\section{Conflict of Interests}

The authors have no known conflict of interests.

\section{References}

[1] D. M. Lloyd-Jones, P. W. F. Wilson, M. G. Larson et al., "Lifetime risk of coronary heart disease by cholesterol levels at selected ages," Archives of Internal Medicine, vol. 163, no. 16, pp. 19661972, 2003.

[2] E. S. Ford and S. Capewell, "Coronary heart disease mortality among young adults in the U.S. from 1980 through 2002: concealed leveling of mortality rates," Journal of the American College of Cardiology, vol. 50, no. 22, pp. 2128-2132, 2007.

[3] A. Lleras-Muney, "The relationship between education and adult mortality in the United States," Review of Economic Studies, vol. 72, no. 1, pp. 189-221, 2005.

[4] E. B. Loucks, J. W. Lynch, L. Pilote et al., "Life-course socioeconomic position and incidence of coronary heart disease: The Framingham Offspring Study," American Journal of Epidemiology, vol. 169, no. 7, pp. 829-836, 2009.

[5] G. A. Kaplan and J. E. Keil, "Socioeconomic factors and cardiovascular disease: a review of the literature," Circulation, vol. 88, no. 4, pp. 1973-1998, 1993.

[6] M. A. González, F. R. Artalejo, and J. D. R. Calero, "Relationship between socioeconomic status and ischaemic heart disease in cohort and case-control studies: 1960-1993," International Journal of Epidemiology, vol. 27, no. 3, pp. 350-358, 1998.
[7] S. Kanjilal, E. W. Gregg, Y. J. Cheng et al., "Socioeconomic status and trends in disparities in 4 major risk factors for cardiovascular disease among US adults, 1971-2002," Archives of Internal Medicine, vol. 166, no. 21, pp. 2348-2355, 2006.

[8] N. A. Christakis and J. H. Fowler, "The collective dynamics of smoking in a large social network," The New England Journal of Medicine, vol. 358, no. 21, pp. 2249-2258, 2008.

[9] N. A. Christakis and J. H. Fowler, "The spread of obesity in a large social network over 32 years," The New England Journal of Medicine, vol. 357, no. 4, pp. 370-379, 2007.

[10] K. M. Emmons, E. M. Barbeau, C. Gutheil, J. E. Stryker, and A. M. Stoddard, "Social influences, social context, and health behaviors among working-class, multi-ethnic adults," Health Education and Behavior, vol. 34, no. 2, pp. 315-334, 2007.

[11] G. Sorensen, E. Barbeau, M. K. Hunt, and K. Emmons, "Reducing social disparities in Tobacco use: a social-contextual model for reducing Tobacco use among Blue-Collar workers," American Journal of Public Health, vol. 94, no. 2, pp. 230-239, 2004.

[12] K. M. Emmons, "Health behaviors in a social context," in Social Epidemiology, L. F. Berkman and I. Kawachi, Eds., pp. 242-266, Oxford University Press, New York, NY, USA, 2000.

[13] F. B. Hu, J. E. Manson, M. J. Stampfer et al., "Diet, lifestyle, and the risk of type 2 diabetes mellitus in women," The New England Journal of Medicine, vol. 345, no. 11, pp. 790-797, 2001.

[14] M. J. Stampfer, F. B. Hu, J. E. Manson, E. B. Rimm, and W. C. Willett, "Primary prevention of coronary heart disease in women through diet and lifestyle," The New England Journal of Medicine, vol. 343, no. 1, pp. 16-22, 2000.

[15] J. Sattelmair, J. Pertman, E. L. Ding, H. W. Kohl, W. Haskell, and I.-M. Lee, "Dose response between physical activity and risk of coronary heart disease: a meta-analysis," Circulation, vol. 124, no. 7, pp. 789-795, 2011.

[16] J. Critchley and S. Capewell, "Smoking cessation for the secondary prevention of coronary heart disease," Cochrane Database of Systematic Reviews, no. 1, Article ID CD003041, 2004.

[17] L. Hartley, E. Igbinedion, J. Holmes et al., "Increased consumption of fruit and vegetables for the primary prevention of cardiovascular diseases," Cochrane Database of Systematic Reviews, vol. 4, no. 6, Article ID CD009874, 2013.

[18] E. Manrique-Garcia, A. Sidorchuk, J. Hallqvist, and T. Moradi, "Socioeconomic position and incidence of acute myocardial infarction: a meta-analysis," Journal of Epidemiology and Community Health, vol. 65, no. 4, pp. 301-309, 2011.

[19] N. Krieger, D. R. Williams, and N. E. Moss, "Measuring social class in us public health research: concepts, methodologies, and guidelines," Annual Review of Public Health, vol. 18, pp. 341-378, 1997.

[20] R. A. Carleton, T. M. Lasater, A. R. Assaf et al., "The Pawtucket Heart Health Program: community changes in cardiovascular risk factors and projected disease risk," American Journal of Public Health, vol. 85, no. 6, pp. 777-785, 1995.

[21] R. V. Luepker, D. M. Murray, D. R. Jacobs Jr. et al., "Community education for cardiovascular disease prevention: Risk factor changes in the Minnesota Heart Health Program," American Journal of Public Health, vol. 84, no. 9, pp. 1383-1393, 1994.

[22] J. W. Farquhar, S. P. Fortmann, J. A. Flora et al., "Effects of communitywide education on cardiovascular disease risk factors. The Stanford Five-City Project," Journal of the American Medical Association, vol. 264, no. 3, pp. 359-365, 1990. 
[23] J. Stamler and J. D. Neaton, "The multiple risk factor intervention trial (MRFIT) -importance then and now: commentary," Journal of the American Medical Association, vol. 300, no. 11, pp. 1343-1345, 2008.

[24] S. E. Chiuve, M. L. McCullough, F. M. Sacks, and E. B. Rimm, "Healthy lifestyle factors in the primary prevention of coronary heart disease among men: benefits among users and nonusers of lipid-lowering and antihypertensive medications," Circulation, vol. 114, no. 2, pp. 160-167, 2006.

[25] M. Pennant, C. Davenport, S. Bayliss, W. Greenheld, T. Marshall, and C. Hyde, "Community programs for the prevention of cardiovascular disease: a systematic review," American Journal of Epidemiology, vol. 172, no. 6, pp. 501-516, 2010.

[26] S. Ebrahim, F. Taylor, K. Ward, A. Beswick, M. Burke, and G. Davey Smith, "Multiple risk factor interventions for primary prevention of coronary heart disease," Cochrane Database of Systematic Reviews, vol. 1, no. 1, Article ID CD001561, 2011.

[27] N. Everage, C. Linkletter, A. Gjelsvik, S. McGarvey, and E. Loucks, "Implementation of permutation testing to determine clustering of social and behavioral risk factors for coronary heart disease NHANES, 2001-2004," Annals of Epidemiology, vol. 23, no. 7, pp. 381-387, 2013.

[28] A. J. Lusis, A. D. Attie, and K. Reue, "Metabolic syndrome: from epidemiology to systems biology," Nature Reviews Genetics, vol. 9, no. 11, pp. 819-830, 2008.

[29] S. M. Grundy, J. I. Cleeman, S. R. Daniels et al., "Diagnosis and management of the metabolic syndrome: an American Heart Association/National Heart, Lung, and Blood Institute scientific statement," Circulation, vol. 112, no. 17, pp. 2735-2752, 2005.

[30] J. Sundström, U. Risérus, L. Byberg, B. Zethelius, H. Lithell, and L. Lind, "Clinical value of the metabolic syndrome for long term prediction of total and cardiovascular mortality: Prospective, population based cohort study," British Medical Journal, vol. 332, no. 7546, pp. 878-882, 2006.

[31] J. Sundström, E. Vallhagen, U. Risérus et al., "Risk associated with the metabolic syndrome versus the sum of its individual components," Diabetes Care, vol. 29, no. 7, pp. 1673-1674, 2006.

[32] P. W. F. Wilson, R. B. D’Agostino, H. Parise, L. Sullivan, and J. B. Meigs, "Metabolic syndrome as a precursor of cardiovascular disease and type 2 diabetes mellitus," Circulation, vol. 112, no. 20, pp. 3066-3072, 2005.

[33] G. Bruno, F. Merletti, A. Biggeri et al., "Metabolic syndrome as a predictor of all-cause and cardiovascular mortality in type 2 diabetes: The Casale Monferrato study," Diabetes Care, vol. 27, no. 11, pp. 2689-2694, 2004.

[34] G. R. Warnick, "Enzymatic methods for quantification of lipoprotein lipids," Methods in Enzymology, vol. 129, pp. 101-123, 1986.

[35] G. R. Warnick, J. Benderson, and J. J. Albers, "Dextran sulfate$\mathrm{Mg} 2+$ precipitation procedure for quantitation of high-densitylipoprotein cholesterol," Clinical Chemistry, vol. 28, no. 6, pp. 1379-1388, 1982.

[36] P. S. Bachorik and J. J. Albers, "Precipitation methods for quantification of lipoproteins," Methods in Enzymology, vol. 129, pp. 78-100, 1986.

[37] Expert Panel on Detection, Evaluation, and Treatment of High Blood Cholesterol in Adults, "Executive summary of the third report of the National Cholesterol Education Program (NCEP) expert panel on detection, evaluation, and treatment of high blood cholesterol in adults (adult treatment panel III)," Journal of the American Medical Association, vol. 285, no. 19, pp. 24862497, 2001.
[38] Physician Examination Procedures Manual [Internet], 2004, http://www.cdc.gov/nchs/data/nhanes/nhanes_05_06/PE.pdf.

[39] A. V. Chobanian, G. L. Bakris, H. R. Black et al., "The seventh report of the joint national committee on prevention, detection, evaluation, and treatment of high blood pressure: the JNC 7 report," Journal of the American Medical Association, vol. 289, no. 19, pp. 2560-2571, 2003.

[40] "Anthropometry Procedures Manual [Internet]," 2002, http://www.cdc.gov/nchs/data/nhanes/nhanes_01_02/body_ measures_year_3.pdf.

[41] National Institutes of Health, National Heart, Lung, and Blood Institute, "Clinical guidelines on the identification, evaluation, and treatment of overweight and obesity in adults: the evidence report," Obesity Research, vol. 6, supplement 2, pp. S51-S210, 1998.

[42] M. W. Slein, G. T. Cori, and C. F. Cori, "A comparative study of hexokinase from yeast and animal tissues," The Journal of Biological Chemistry, vol. 186, no. 2, pp. 763-779, 1950.

[43] R. R. Pate, M. Pratt, S. N. Blair et al., "Physical activity and public health: a recommendation from the Centers for Disease Control and Prevention and the American College of Sports Medicine," Journal of the American Medical Association, vol. 273, no. 5, pp. 402-407, 1995.

[44] W. L. Haskell, I.-M. Lee, R. R. Pate et al., "Physical activity and public health: updated recommendation for adults from the American College of Sports Medicine and the American Heart Association," Circulation, vol. 116, no. 9, pp. 1081-1093, 2007.

[45] SP Questionnaire Component: Physical Activity Questionnaire Data [Internet], 2005, http://www.cdc.gov/nchs/data/nhanes/ nhanes_03_04/paq_c.pdf.

[46] "SP Questionnaire Component: Physical Activity Individual Activities Data [Internet]," 2005, http://www.cdc.gov/nchs/data /nhanes/nhanes_03_04/paqiaf_c.pdf.

[47] What we Eat in America, NHANES [Internet], 2010, http:// www.ars.usda.gov/Services/docs.htm?docid=13793.

[48] NHANES-documentation, Codebook, and Frequenciesdietary interview—individual foods (First Day) - 2003-2004 [Internet], 2007, http://www.cdc.gov/nchs/data/nhanes/nhanes _03_04/drliff_c.pdf.

[49] Food Surveys Products and Services- what we Eat in America [Internet], 2010, http://www.ars.usda.gov/Services/docs. htm?docid=13793.

[50] S. Welsh, C. Davis, and A. Shaw, "Development of the food guide pyramid," Nutrition Today, vol. 26, no. 6, pp. 12-23, 1992.

[51] "Explaining the Rise in Educational Gradients in Mortality," NBER Working Paper 15678, 2010, http://www.nber.org/ papers/w15678.pdf.

[52] J. K. Montez, M. D. Hayward, D. C. Brown, and R. A. Hummer, "Why is the educational gradient of mortality steeper for men?" Journals of Gerontology B, vol. 64, no. 5, pp. 625-634, 2009.

[53] T. N. Akbaraly, M. Kivimaki, M.-L. Ancelin et al., "Metabolic syndrome, its components, and mortality in the elderly," Journal of Clinical Endocrinology and Metabolism, vol. 95, no. 11, pp. E327-E332, 2010.

[54] A. Galassi, K. Reynolds, and J. He, "Metabolic syndrome and risk of cardiovascular disease: a meta-analysis," American Journal of Medicine, vol. 119, no. 10, pp. 812-819, 2006.

[55] E. B. Loucks, M. Abrahamowicz, Y. Xiao, and J. W. Lynch, "Associations of education with 30 year life course blood pressure trajectories: Framingham Offspring Study," BMC Public Health, vol. 11, article 139, 2011. 
[56] R. C. Thurston, L. D. Kubzansky, I. Kawachi, and L. F. Berkman, "Is the association between socioeconomic position and coronary heart disease stronger in women than in men?" American Journal of Epidemiology, vol. 162, no. 1, pp. 57-65, 2005.

[57] M. Alonso González, F. Rodríguez Artalejo, and J. Del Rey Calero, "Relationship between socioeconomic status and ischaemic heart disease in cohort and case-control studies: 1960-1993," International Journal of Epidemiology, vol. 27, no. 3, pp. 350-358, 1998.

[58] H. M. Colhoun, H. Hemingway, and N. R. Poulter, "Socioeconomic status and blood pressure: an overview analysis," Journal of Human Hypertension, vol. 12, no. 2, pp. 91-110, 1998.

[59] R. Chen and H. Tunstall-Pedoe, "Socioeconomic deprivation and waist circumference in men and women: the Scottish MONICA surveys 1989-1995," European Journal of Epidemiology, vol. 20, no. 2, pp. 141-147, 2005.

[60] A. F. Brown, S. L. Ettner, J. Piette et al., "Socioeconomic position and health among persons with diabetes mellitus: a conceptual framework and review of the literature," Epidemiologic Reviews, vol. 26, pp. 63-77, 2004.

[61] P. Muennig, N. Sohler, and B. Mahato, "Socioeconomic status as an independent predictor of physiological biomarkers of cardiovascular disease: Evidence from NHANES," Preventive Medicine, vol. 45, no. 1, pp. 35-40, 2007.

[62] G. A. Mensah, A. H. Mokdad, E. S. Ford, K. J. Greenlund, and J. B. Croft, "State of disparities in cardiovascular health in the United States," Circulation, vol. 111, no. 10, pp. 1233-1241, 2005.

[63] L. Erhardt, "Cigarette smoking: an undertreated risk factor for cardiovascular disease," Atherosclerosis, vol. 205, no. 1, pp. 2332, 2009.

[64] W. Y. Craig, G. E. Palomaki, and J. E. Haddow, "Cigarette smoking and serum lipid and lipoprotein concentrations: an analysis of published data," British Medical Journal, vol. 298, no. 6676, pp. 784-788, 1989.

[65] S. C. Campbell, R. J. Moffatt, and B. A. Stamford, "Smoking and smoking cessation-the relationship between cardiovascular disease and lipoprotein metabolism: a review," Atherosclerosis, vol. 201, no. 2, pp. 225-235, 2008.

[66] A. Chiolero, D. Faeh, F. Paccaud, and J. Cornuz, "Consequences of smoking for body weight, body fat distribution, and insulin resistance," American Journal of Clinical Nutrition, vol. 87, no. 4, pp. 801-809, 2008.

[67] R. H. Fagard, "Smoking amplifies cardiovascular risk in patients with hypertension and diabetes," Diabetes Care, vol. 32, pp. S429-S431, 2009.

[68] D. Haire-Joshu, R. E. Glasgow, and T. L. Tibbs, "Smoking and diabetes," Diabetes Care, vol. 22, no. 11, pp. 1887-1898, 1999.

[69] A. Ascherio, C. Hennekens, W. C. Willett et al., "Prospective study of nutritional factors, blood pressure, and hypertension among US women," Hypertension, vol. 27, no. 5, pp. 1065-1072, 1996.

[70] L. J. Appel, "The role of diet in the prevention and treatment of hypertension," Current Atherosclerosis Reports, vol. 2, no. 6, pp. 521-528, 2000.

[71] S. A. McNaughton, G. D. Mishra, A. M. Stephen, and M. E. J. Wadsworth, "Dietary patterns throughout adult life are associated with body mass index, waist circumference, blood pressure, and red cell folate," Journal of Nutrition, vol. 137, no. 1, pp. 99-105, 2007.

[72] E. R. Miller III, T. P. Erlinger, and L. J. Appel, "The effects of macronutrients on blood pressure and lipids: an overview of the
DASH and OmniHeart trials," Current Atherosclerosis Reports, vol. 8, no. 6, pp. 460-465, 2006.

[73] P. Carter, L. J. Gray, J. Troughton, K. Khunti, and M. J. Davies, "Fruit and vegetable intake and incidence of type 2 diabetes mellitus: systematic review and meta-analysis," British Medical Journal, vol. 341, Article ID c4229, 2010.

[74] S. Liu, M. Serdula, S.-J. Janket et al., "A prospective study of fruit and vegetable intake and the risk of type 2 diabetes in women," Diabetes Care, vol. 27, no. 12, pp. 2993-2996, 2004.

[75] M. Hamer and Y. Chida, "Intake of fruit, vegetables, and antioxidants and risk of type 2 diabetes: systematic review and meta-analysis," Journal of Hypertension, vol. 25, no. 12, pp. 23612369, 2007.

[76] F. B. Hu, "Plant-based foods and prevention of cardiovascular disease: an overview," The American Journal of Clinical Nutrition, vol. 78, no. 3, pp. 544S-551S, 2003.

[77] D. J. A. Jenkins, J. M. W. Wong, C. W. C. Kendall et al., "The effect of a plant-based low-carbohydrate ("eco-atkins") diet on body weight and blood lipid concentrations in hyperlipidemic subjects," Archives of Internal Medicine, vol. 169, no. 11, pp. 10461054,2009

[78] B. C. Tohill, J. Seymour, M. Serdula, L. Kettel-Khan, and B. J. Rolls, "What epidemiologic studies tell us about the relationship between fruit and vegetable consumption and body weight," Nutrition Reviews, vol. 62, no. 10, pp. 365-374, 2004.

[79] S. Alinia, O. Hels, and I. Tetens, "The potential association between fruit intake and body weight-a review," Obesity Reviews, vol. 10, no. 6, pp. 639-647, 2009.

[80] D. R. Jacobs Jr., M. D. Gross, and L. C. Tapsell, "Food synergy: an operational concept for understanding nutrition," American Journal of Clinical Nutrition, vol. 89, no. 5, pp. 1543S-1548S, 2009.

[81] F. B. Hu and W. C. Willett, "Optimal diets for prevention of coronary heart disease," Journal of the American Medical Association, vol. 288, no. 20, pp. 2569-2578, 2002.

[82] H. I. Katcher, A. M. Hill, J. L. G. Lanford, J. S. Yoo, and P. M. KrisEtherton, "Lifestyle approaches and dietary strategies to lower LDL-cholesterol and triglycerides and raise HDL-cholesterol," Endocrinology and Metabolism Clinics of North America, vol. 38, no. 1, pp. 45-78, 2009.

[83] S. P. Whelton, A. Chin, X. Xin, and J. He, "Effect of aerobic exercise on blood pressure: a meta-analysis of randomized, controlled trials," Annals of Internal Medicine, vol. 136, no. 7, pp. 493-503, 2002.

[84] R. H. Fagard and V. A. Cornelissen, "Effect of exercise on blood pressure control in hypertensive patients," European Journal of Cardiovascular Prevention and Rehabilitation, vol. 14, no. 1, pp. 12-17, 2007.

[85] K. Ohkawara, S. Tanaka, M. Miyachi, K. Ishikawa-Takata, and I. Tabata, "A dose-response relation between aerobic exercise and visceral fat reduction: Systematic review of clinical trials," International Journal of Obesity, vol. 31, no. 12, pp. 1786-1797, 2007.

[86] S. J. Kay and M. A. Fiatarone Singh, "The influence of physical activity on abdominal fat: a systematic review of the literature," Obesity Reviews, vol. 7, no. 2, pp. 183-200, 2006.

[87] D. Canoy and I. Buchan, "Challenges in obesity epidemiology," Obesity Reviews, vol. 8, no. 1, pp. 1-11, 2007.

[88] M. Fogelholm and K. Kukkonen-Harjula, "Does physical activity prevent weight gain - a systematic review," Obesity Reviews, vol. 1, no. 2, pp. 95-111, 2000. 
[89] W. H. M. Saris, S. N. Blair, M. A. van Baak et al., "How much physical activity is enough to prevent unhealthy weight gain? Outcome of the IASO 1st stock conference and consensus statement," Obesity Reviews, vol. 4, no. 2, pp. 101-114, 2003.

[90] J. Tuomilehto, J. Lindström, J. G. Eriksson et al., "Prevention of type 2 diabetes mellitus by changes in lifestyle among subjects with impaired glucose tolerance," The New England Journal of Medicine, vol. 344, no. 18, pp. 1343-1350, 2001.

[91] R. J. Sigal, G. P. Kenny, D. H. Wasserman, C. CastanedaSceppa, and R. D. White, "Physical activity/exercise and type 2 diabetes: a consensus statement from the American Diabetes Association," Diabetes Care, vol. 29, no. 6, pp. 1433-1438, 2006.

[92] S. Kodama, S. Tanaka, K. Saito et al., "Effect of aerobic exercise training on serum levels of high-density lipoprotein cholesterol: a meta-analysis," Archives of Internal Medicine, vol. 167, no. 10, pp. 999-1008, 2007.

[93] P. Kokkinos and J. Myers, "Exercise and physical activity: clinical outcomes and applications," Circulation, vol. 122, no. 16, pp. 1637-1648, 2010.

[94] E. B. Loucks, K. T. Magnusson, S. Cook, D. H. Rehkopf, E. S. Ford, and L. F. Berkman, "Socioeconomic position and the metabolic syndrome in early, middle, and late life: evidence from NHANES 1999-2002," Annals of Epidemiology, vol. 17, no. 10, pp. 782-790, 2007.

[95] E. B. Loucks, D. H. Rehkopf, R. C. Thurston, and I. Kawachi, "Socioeconomic disparities in metabolic syndrome differ by gender: evidence from NHANES III," Annals of Epidemiology, vol. 17, no. 1, pp. 19-26, 2007.

[96] B. T. Smith, J. W. Lynch, C. S. Fox et al., "Life-course socioeconomic position and type 2 diabetes mellitus the Framingham offspring study," American Journal of Epidemiology, vol. 173, no. 4, pp. 438-447, 2011.

[97] E. Agardh, P. Allebeck, J. Hallqvist, T. Moradi, and A. Sidorchuk, "Type 2 diabetes incidence and socio-economic position: A systematic review and meta-analysis," International Journal of Epidemiology, vol. 40, no. 3, pp. 804-818, 2011.

[98] E. A. Finkelstein, C. J. Ruhm, and K. M. Kosa, "Economic causes and consequences of obesity," Annual Review of Public Health, vol. 26, pp. 239-257, 2005.

[99] L. S. Kinder, M. R. Carnethon, L. P. Palaniappan, A. C. King, and S. P. Fortmann, "Depression and the metabolic syndrome in young adults: findings from the Third National Health and Nutrition Examination Survey," Psychosomatic Medicine, vol. 66, no. 3, pp. 316-322, 2004.

[100] J. M. McCaffery, R. Niaura, J. F. Todaro, G. E. Swan, and D. Carmelli, "Depressive symptoms and metabolic risk in adult male twins enrolled in the National Heart, Lung, and Blood Institute Twin Study," Psychosomatic Medicine, vol. 65, no. 3, pp. 490-497, 2003.

[101] J. Dallongeville, D. Cottel, J. Ferrières et al., "Household income is associated with the risk of metabolic syndrome in a sexspecific manner," Diabetes Care, vol. 28, no. 2, pp. 409-415, 2005.

[102] Y.-W. Park, S. Zhu, L. Palaniappan, S. Heshka, M. R. Carnethon, and S. B. Heymsfield, "The metabolic syndrome: prevalence and associated risk factor findings in the US population from the Third National Health and Nutrition Examination Survey, 1988-1994," Archives of Internal Medicine, vol. 163, no. 4, pp. 427-436, 2003.

[103] K. M. Emmons, C. M. McBride, E. Puleo et al., "Prevalence and predictors of multiple behavioral risk factors for colon cancer," Preventive Medicine, vol. 40, no. 5, pp. 527-534, 2005.
[104] G. Biró, K. F. A. M. Hulshof, L. Ovesen, and J. A. Amorim Cruz, "Selection of methodology to assess food intake," European Journal of Clinical Nutrition, vol. 56, no. 2, pp. S25-S32, 2002.

[105] NHANES, 2001-2002-Manuals, Brochures, and Consent Documents, 2010, http://www.cdc.gov/nchs/nhanes/nhanes20012002/current_nhanes_01_02.htm. 


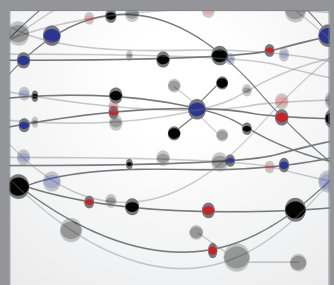

The Scientific World Journal
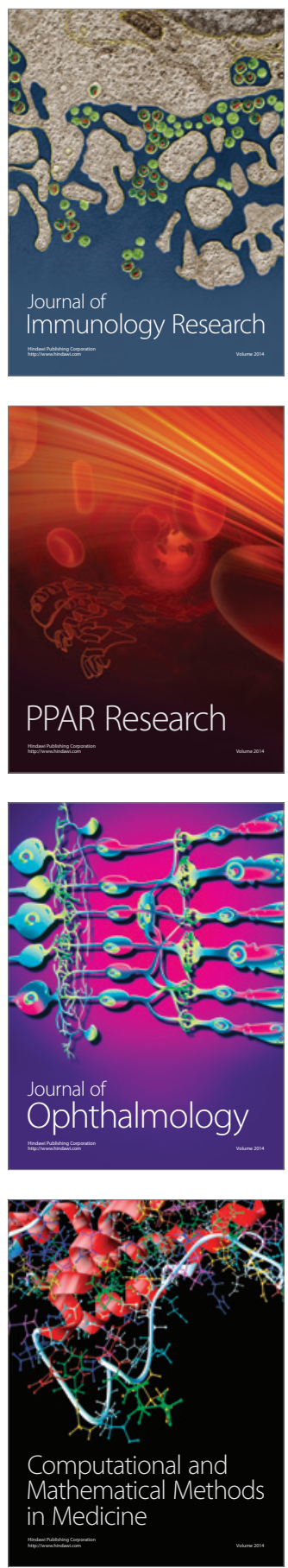

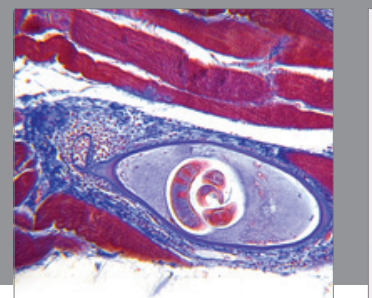

Gastroenterology

Research and Practice
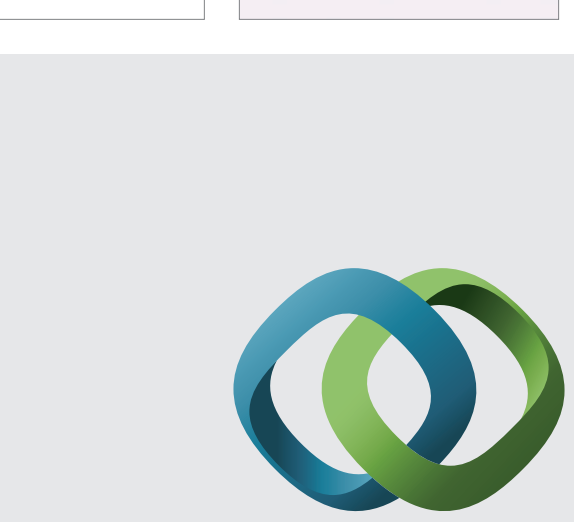

\section{Hindawi}

Submit your manuscripts at

http://www.hindawi.com
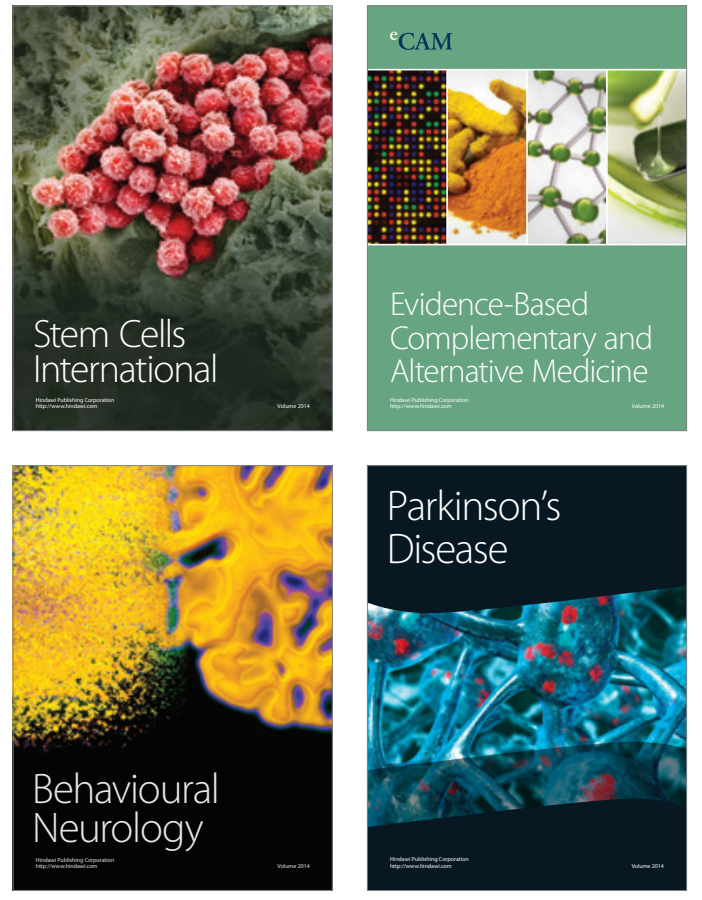


Disease Markers
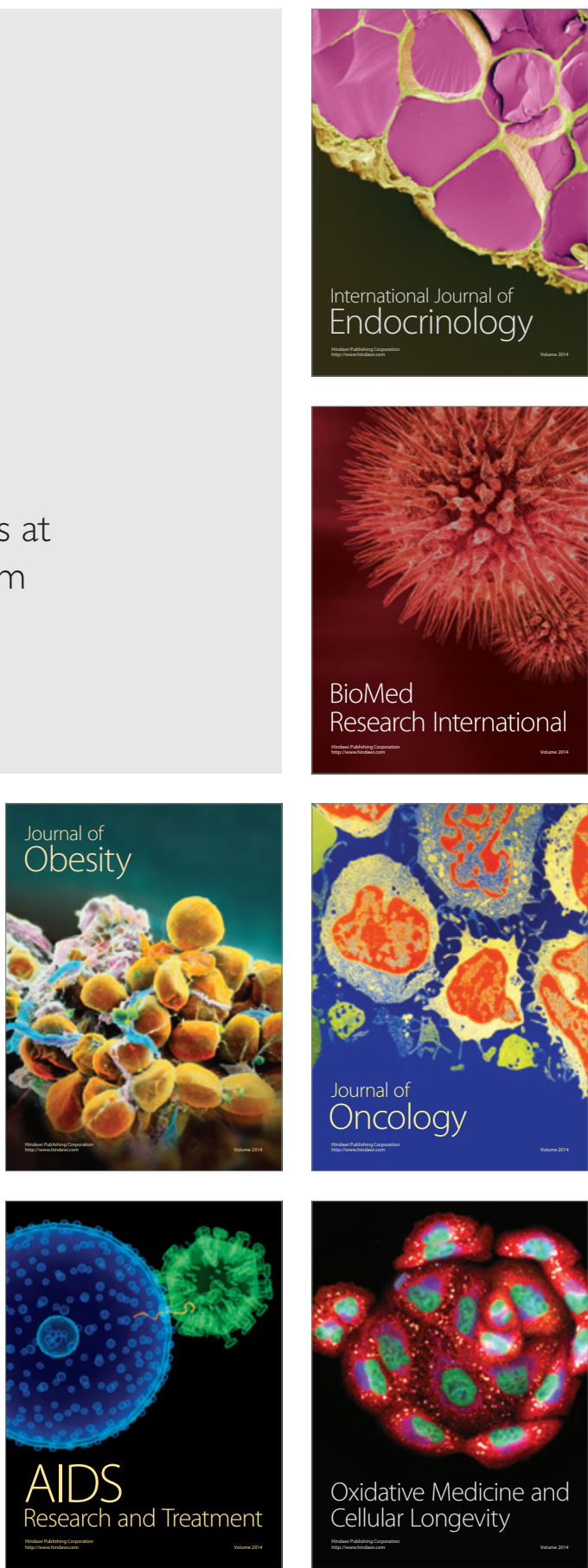